\title{
Met and Unmet Needs in an Inpatient Psychiatry Setting in Spain
}

\author{
Carlos Gomez-Sanchez- \\ Lafuente (D) ${ }^{1,2}$ \\ Jose Guzman-Parra' \\ Maria Alejandra Lopez- \\ Zambrano' \\ Berta Moreno-Kustner ${ }^{2}$ \\ Fermin Mayoral-Cleries (D) \\ 'Unidad de Gestión Clínica de Salud \\ Mental, Instituto de Investigación \\ Biomédica de Málaga (IBIMA), Hospital \\ Regional Universitario de Málaga, Málaga, \\ 29010, Spain; ${ }^{2}$ Universidad de Málaga, \\ Andalucía Tech, Facultad de Psicología, \\ Málaga, 2907I, Spain
}

Purpose: Patients with severe mental disorder have health and social needs that require care. The aim of the study was to determine the main areas of perceived needs among inpatients with severe mental disorders and to identify risk factors of the group of patients with the greatest unmet needs.

Patients and Methods: A total of 150 patients with severe mental illness were assessed during admission prior to discharge from the hospital. Camberwell Assessment of Needs was used as evaluation measure, in addition to clinical status (The Brief Psychiatric Rating Scale), social functioning (Personal and Social Performance) and sociodemographic variables. A descriptive and a multivariate logistic regression analysis were used to analyse variables related to the group of patients with the highest number of unmet needs $(\geq 3)$.

Results: Mean number of needs was 7.93, being 4.61 the mean number of needs met and 3.32 the needs unmet. The highest proportion of unmet needs were intimate relationships $(44.0 \%)$, company $(40.7 \%)$ and daytime activities (38.7\%). A relationship was also found between the presence of three or more unmet needs and the following variables: Brief Psychiatric Rating Scale score $(p=0.004)$, Personal and Social Performance score $(p=0.013)$, marital status $(\mathrm{p}=0.018)$, employment status $(\mathrm{p}=0.009)$ and voluntary admission $(\mathrm{p}=0.032)$. The multivariate model explained $29.5 \%$ of the variance (Nagelkerke's $\mathrm{R}^{2}: 0.295$ ).

Conclusion: Treatments aimed at improving social relationships and daytime activities could be a good option for inpatients with many unmet needs.

Keywords: needs assessment, severe mental illness, inpatient, functioning

\section{Introduction}

Severe mental disorders involve a combination of health, emotional and social needs that require care. Needs have been defined as the requirement of the individual patient to enable him to achieve, maintain or restore an acceptable level of social independence, and quality of life. ${ }^{1}$ A need is unmet when the patient does not receive adequate help or when an intervention has no effect; a need is met when the help provided to the individual is effective. ${ }^{2}$ Addressing needs systematically by psychiatrists seems to improve quality of life and reducing unmet needs. ${ }^{3,4}$ Better relationships with informal care networks have been associated with reducing informal needs and use of hospital services. ${ }^{5}$ Therefore, finding the most important areas and factors influencing the maintenance of unmet needs is key to design better personalised interventions and to improve services according to the real needs of psychiatry inpatients. ${ }^{2,6,7}$

Needs have been assessed in different contexts, In community-based samples, needs related to the illness (ie, psychological distress, psychotic symptoms)
Correspondence: Carlos GomezSanchez-Lafuente

Plaza Hospital Civil S/N Unidad de Investigación Salud Mental, Planta I

Pabellón 4, Malaga, 290I0, Spain

Tel +0034951290307

Email gomisanchezlafuente@gmail.com 
and social and emotional needs, such as company and daytime activities are the most prevalent. ${ }^{8,9}$ Patients in hospital settings perceive a higher number of needs, including intimate relations, sexual needs and information about the treatment. ${ }^{10-13}$ The presence of unmet needs in psychiatric inpatients has been associated with an increased severity of symptoms, ${ }^{13}$ social impairment, functional disability and greater negative perception of the illness. ${ }^{14,15}$ On the other hand, meeting needs have been associated with an improved motivation for treatment, ${ }^{16}$ subjective welfare ${ }^{17}$ and better subjective quality of life after hospitalization. ${ }^{18-20}$ Individuals who frequently visit psychiatric emergency rooms most common needs were psychological distress, psychotic symptoms, daytime activities and intimate relationships in Sweden. ${ }^{21}$ Unmet needs related to safety to self and others, substance use, and psychotic symptoms were significantly associated with repeated emergency department visits. $^{22}$

Needs also show differences depending on the region. The European Psychiatric Services: Inputs Linked to Outcomes and Needs (EPSILON) study compared patients' needs in five European cities. Unmet needs were higher in big urban areas. ${ }^{23}$ Patients in rural settings had fewer needs than those in an inner-city area in London. ${ }^{24}$ One study found that household, money management and preparing meals needs were higher in individuals with psychosis in south of Spain, whereas symptoms and behavioural problems were more prevalent in Germany. ${ }^{25}$ The mean number of perceived needs was higher in countries with lower resources or going through an economic crisis, such as Greece, Latin America and India. $^{26-28}$

Hospitalisation for people with mental illness changed in Spain after the deinstitutionalization movement started in the $1980 \mathrm{~s} .{ }^{25}$ Since then, community-based care and the development of units and programmes for outpatient care, home care and partial hospitalisation have been prioritised. $^{29}$ Length of hospital stay has been reduced and admissions occur when the community care has not been effective. ${ }^{30-32}$ Some studies have analysed outpatients needs in Spain, ${ }^{8,10}$ but psychiatric inpatients needs remain to be investigated. The aim of this study was to determine the main areas of self-reported needs among inpatients with severe mental disorder and to assess risk factors of the group of patients with the greatest number of unmet needs.

\section{Patients and Methods}

\section{Study Settings}

This study was set in an inpatient unit of the University Regional Hospital of Malaga. The unit provides mental health hospitalization services for a catchment area of approximately 570,000 inhabitants. The unit has 42 beds and an average of 846 annual hospital admissions (1998-2019 data). This is the only public inpatient unit in the area of reference. The unit is part of the system of care for community mental health belonging to the Andalusian Health Service (SAS), which provides universal health coverage to anyone living in the region. The Malaga Northeast Ethics Committee approved the study. All participants gave informed consent to participate.

\section{Study Design}

This was a cross-sectional study. Patients were evaluated during admission prior to discharge from the hospital. Inclusion criteria were individuals older than 18 and fluent in Spanish admitted to the inpatient unit. Exclusion criteria were as follows: patients with no permanent address or a permanent address outside the catchment area, patients with mental retardation and patients whose primary diagnosis was substance use disorder. Participants were included in the study if they were able to complete the assessment and sign the informed consent. The medical staff were informed about characteristics of the study and were asked to notify the researchers if the patient was eligible for the study and agreed to participate. The researchers then evaluated whether the patient met the inclusion and exclusion criteria. The assessment was conducted by psychologists or psychiatrists with clinical experience who were previously trained to administer evaluation instruments. The assessment was performed prior to discharge from the hospital in all cases. Users had to be clinically stable of the symptomatology that motivated the admission. The evaluation period was from February 2009 to September 2010. Out of 780 patients admitted to hospital during the period, 439 belonged to the reference catchment area. The evaluators were available 2 days per week and for this reason participation was not offered to all eligible patients. One hundred and fifty patients met the inclusion criteria and consented to participate. Diagnosis was made by specialist psychiatrists following the ICD-10 criteria. 


\section{Measures}

\section{Camberwell Assessment of Needs (CAN Research Version) $)^{33}$}

This instrument evaluates the global needs of people with mental disorders in the month before the evaluation. The evaluation consists of two parts, one for the patient and one for the professional in charge of the patient. The instrument assesses 22 domains of needs including those related to clinical features, family, self-care and use of social services. In this study, we used the patient version of the instrument. A need is identified if an individual has any serious problem in a specific domain. If the inpatient perceives there is a current problem despite receiving help, that is rated as Unmet Need. Otherwise, if the individual is receiving help for that issue and the problem is reducing to a moderate level or less, the need rating is Met Need. The instrument was validated in Spanish and had adequate reliability and validity parameters. ${ }^{34}$

\section{Personal and Social Performance (PSP) ${ }^{35}$}

This is a validated clinician-rated scale designed to measure patients' functioning in four areas: (a) personal and social relationships, (b) socially useful activities, (c) selfcare, and (d) disturbing and aggressive behaviours. Impairment in each area was rated on a 6-point scale, being $1=$ absent and $6=$ very severe. In addition to the score in each domain, a total score of 0 to 100 points can be obtained. An overall score of 71-100 points indicates mild or no difficulty, 31-70 medium difficulty and under 31 serious difficulties needing intense support. The Spanish version shows adequate validity and reliability parameters in different samples. ${ }^{36}$

\section{Brief Psychiatric Rating Scale (BPRS) ${ }^{37}$}

This scale is used for evaluating the severity of psychopathological symptoms in psychotic patients. We used the Spanish 24-items version with possible answers ranging from 1 to $7 .^{38}$

Sociodemographic and clinical variables: age, gender, marital status, type of cohabitation, educational level, employment status, length of stay, number of prior admissions, legal status of admission (voluntary or involuntary), type of admission (programmed or urgent) and diagnosis (ICD-10).

\section{Statistical Analysis}

A descriptive analysis using frequency tables examined main needs, satisfied needs, unmet needs and a ratio between the presence of a need/need not covered of inpatients. The percentage of participants that needed moderate or high help from services for each need, frequency of satisfaction, frequency of adequate help and percentage of participants who received moderate or high help from informal networks and services were also described. Bivariate logistic regression analysis was performed to determine factors associated with having three or more unmet needs (Median). A multivariate logistic regression analysis with the variables that were statistically significant $(p<0.05)$ in the bivariate analysis was conducted. Since PSP and BPRS scores showed high collinearity, we decided to enter only BPRS score in the multivariate model due to the fact that it showed a stronger statistically association in the bivariate analysis. The significance level was set at 0.05 (two-sided). $\mathrm{R}$ version 3.4 .1 and $\mathrm{R}$ Commander package were used for the analysis.

\section{Results}

Mean age of the sample was 42.54 years $(\mathrm{SD}=12.57)$, and $58.6 \%$ were male. Median length of stay was 12 days (interquartile Range $=14$ ). Time from admission to needs evaluation had a median of 11 days (interquartile range $=$ 14). The majority of the sample had an involuntary admission $(78.5 \%)$. Patients had a low level of education, with $60.7 \%$ having no studies or just primary education. Table 1 includes demographic characteristics of the sample.

Mean number of needs was 7.93 , being 4.61 the mean number of needs met and 3.32 the needs unmet. The highest percentage of needs was found in these CAN domains: psychological distress $(63.3 \%)$, daytime activities $(60.7 \%)$ and information $(60.0 \%)$. The highest proportion of unmet needs were intimate relationships (44.0\%), company (40.7\%) and daytime activities (38.7\%). Regarding the ratio between perceived needs and unmet needs, that may indicate possible areas of needs that are not adequately provided, sexual expression (95\%), personal relationships (94\%), and company (76\%) highlighted. Table 2 presents met and unmet needs.

Psychological distress (36.7\%), intimate relationships $(34.0 \%)$ and information (30.0\%) were the three main areas that patients perceived that they needed moderate or high help from services. The areas with less satisfaction with the help received were company (63.3\%), intimate relationships (63.3\%) and daytime activities (63.3\%). More information about satisfaction and appropriateness of help received, subjective perception of help from services and informal networks is shown in Table 3. 
Table I Demographic and Clinical Variables of the Sample

\begin{tabular}{|c|c|c|c|c|c|}
\hline Sample $(N=150)$ & Total & $\begin{array}{c}<3 \text { Unmet Needs } \\
(\mathbf{N}=\mathbf{7 2})\end{array}$ & $\begin{array}{c}\geq 3 \text { Unmet Needs } \\
(\mathbf{N}=\mathbf{7 8})\end{array}$ & OR & $\mathbf{p}$ \\
\hline Age, Mean (SD) & $42.54(12.57)$ & $44.00(17)$ & $42.00(18)$ & 0.993 & 0.618 \\
\hline Length of stay, Median (IQR) & $12(14)$ & $13.00(15)$ & $11.50(15)$ & 0.985 & 0.113 \\
\hline Time from admission to needs evaluation, Median (IQR) & $11(15)$ & $11(15)$ & $10.5(14)$ & 0.988 & 0.242 \\
\hline $\begin{array}{l}\text { Age, N (\%) } \\
\quad \leq 29 \\
30-44 \\
45-59 \\
60+\text { (Reference) }\end{array}$ & $\begin{array}{l}27(18.0) \\
51(34.0) \\
57(38.0) \\
11(7.3)\end{array}$ & $\begin{array}{l}13(18.8) \\
24(34.8) \\
26(37.8) \\
6(8.7 \%)\end{array}$ & $\begin{array}{l}14(18.2) \\
27(35.1) \\
31(40.3) \\
5(6.5 \%)\end{array}$ & $\begin{array}{l}1.292 \\
1.350 \\
1.431\end{array}$ & $\begin{array}{l}0.959 \\
0.721 \\
0.653 \\
0.586\end{array}$ \\
\hline $\begin{array}{l}\text { Sex, } N(\%) \\
\quad \text { Male } \\
\text { Female (Reference) }\end{array}$ & $\begin{array}{l}88(58.6) \\
62(41.4)\end{array}$ & $\begin{array}{l}4 \mid(59.6) \\
31(43 . I)\end{array}$ & $\begin{array}{l}47(60.3) \\
31(39.7)\end{array}$ & $\mathrm{I} .146$ & 0.681 \\
\hline $\begin{array}{l}\text { Marital Status, N (\%) } \\
\text { Married/partnered } \\
\text { Separated/divorced } \\
\text { Single (Reference) }\end{array}$ & $\begin{array}{l}39(26.0) \\
35(23.3) \\
76(50.7)\end{array}$ & $\begin{array}{l}26(36.1) \\
12(16.7) \\
34(47.2)\end{array}$ & $\begin{array}{l}13(16.7) \\
23(29.5) \\
42(53.8)\end{array}$ & $\begin{array}{l}0.405 \\
1.552\end{array}$ & $\begin{array}{l}0.018 \\
0.028 \\
0.301\end{array}$ \\
\hline $\begin{array}{l}\text { Educational Level, N (\%) } \\
\text { Basic education } \\
\text { Primary school } \\
\text { Secondary school } \\
\text { University (Reference) }\end{array}$ & $\begin{array}{l}51(34.0) \\
46(30.7) \\
42(28.0) \\
\text { II }(7.3)\end{array}$ & $\begin{array}{c}27(37.5) \\
22(30.6) \\
20(27.8) \\
3(4.2)\end{array}$ & $\begin{array}{l}24(30.8) \\
24(30.8) \\
22(28.2) \\
8(10.3)\end{array}$ & $\begin{array}{l}0.333 \\
0.409 \\
0.412\end{array}$ & $\begin{array}{l}0.519 \\
0.139 \\
0.226 \\
0.234\end{array}$ \\
\hline $\begin{array}{l}\text { Cohabitation, } \mathrm{N}(\%) \\
\text { Parents } \\
\text { Own family } \\
\text { Other family or friends } \\
\text { Alone (Reference) }\end{array}$ & $\begin{array}{l}55(36.7) \\
39(26.0) \\
25(16.7) \\
31(20.7)\end{array}$ & $\begin{array}{l}25(34.7) \\
23(31.9) \\
15(20.8) \\
9(12.5)\end{array}$ & $\begin{array}{l}30(38.5) \\
16(20.5) \\
10(12.8) \\
22(28.2)\end{array}$ & $\begin{array}{l}0.491 \\
0.285 \\
0.273\end{array}$ & $\begin{array}{l}0.054 \\
0.138 \\
0.014 \\
\mathbf{0 . 0 2 2}\end{array}$ \\
\hline $\begin{array}{l}\text { Employment status, N (\%) } \\
\text { Working } \\
\text { Unemployed } \\
\text { Retired } \\
\text { Pensioner (Reference) }\end{array}$ & $\begin{array}{c}20(13.3) \\
40(26.7) \\
10(6.7) \\
80(53.3)\end{array}$ & $\begin{array}{c}11(15.3) \\
10(13.9) \\
7(9.7) \\
44(6 I .1)\end{array}$ & $\begin{array}{c}9(11.5) \\
30(38.5) \\
3(3.8) \\
36(46.2)\end{array}$ & $\begin{array}{l}1.000 \\
3.667 \\
0.524\end{array}$ & $\begin{array}{c}0.009 \\
1 \\
0.002 \\
0.524\end{array}$ \\
\hline $\begin{array}{l}\text { Legal status of admission }{ }^{\mathrm{a}}, \mathrm{N}(\%) \\
\text { Voluntary } \\
\text { Involuntary (reference) }\end{array}$ & $\begin{array}{c}32(21.5) \\
117(78.0)\end{array}$ & $\begin{array}{l}10(13.9) \\
62(86.1)\end{array}$ & $\begin{array}{l}22(28.6) \\
55(71.4)\end{array}$ & 2.480 & 0.032 \\
\hline $\begin{array}{l}\text { History of hospitalisation, N (\%) } \\
\text { No } \\
\text { Yes (reference) }\end{array}$ & $\begin{array}{c}10(6.7) \\
140(93.3)\end{array}$ & $\begin{array}{c}4(5.6) \\
68(94.4)\end{array}$ & $\begin{array}{c}6(7.7) \\
72(92.3)\end{array}$ & 1.417 & 0.602 \\
\hline $\begin{array}{l}\text { Type of admission, } \mathrm{N}(\%) \\
\text { Programmed } \\
\text { Urgent (reference) }\end{array}$ & $\begin{array}{l}35(23.3) \\
115(76.7)\end{array}$ & $\begin{array}{l}20(27.8) \\
52(72.2)\end{array}$ & $\begin{array}{l}15(19.2) \\
63(80.8)\end{array}$ & 0.619 & 0.218 \\
\hline
\end{tabular}

(Continued) 
Table I (Continued).

\begin{tabular}{|c|c|c|c|c|c|}
\hline Sample $(N=150)$ & Total & $\begin{array}{c}<3 \text { Unmet Needs } \\
(\mathbf{N}=72)\end{array}$ & $\begin{array}{c}\geq 3 \text { Unmet Needs } \\
(\mathbf{N}=78)\end{array}$ & OR & $\mathbf{p}$ \\
\hline Diagnosis, N (\%) & & $26(36.1)$ & $32(41.0)$ & & 0.355 \\
\hline Schizophrenia, schizoaffective or delusional disorder & $58(38.7)$ & $19(26.4)$ & $13(16.7)$ & 1.231 & 0.614 \\
\hline Bipolar disorder & $32(21.3)$ & $7(9.7)$ & $13(16.7)$ & 0.648 & 0.428 \\
\hline Personality disorder & $20(13.3)$ & $20(27.8)$ & $20(25.6)$ & 1.827 & 0.274 \\
\hline Other disorder (Reference) & $40(26.7)$ & & & & \\
\hline PSP, mean (SD) & $40.33(20.58)$ & $44.75(20.69)$ & $36.24(19.74)$ & 0.979 & 0.013 \\
\hline \multicolumn{6}{|l|}{ BPRS, mean (SD) } \\
\hline Total & $42.88(13.11)$ & $39.56(10.42)$ & $45.94(14.58)$ & 1.044 & 0.004 \\
\hline Positive symptoms & $14.59(7.45)$ & $13.29(6.42)$ & $15.78(8.15)$ & 1.048 & 0.044 \\
\hline Depressive symptoms & $12.32(4.86)$ & $10.83(4.29)$ & |3.7| (4.98) & 1.155 & 0.001 \\
\hline Negative symptoms & $7.62(3.6 I)$ & $7.53(3.69)$ & 7.71 (3.56) & 1.014 & 0.763 \\
\hline Manic symptoms & $10.52(4.4 I)$ & $9.94(3.96)$ & II.05 (4.75) & 1.061 & 0.129 \\
\hline Disorientation & $2.66(1.38)$ & $2.50(1.17)$ & $2.81(1.55)$ & 1.183 & 0.179 \\
\hline
\end{tabular}

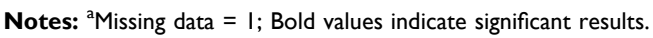

Abbreviations: OR, odds ratio; PSP, personal and social performance scale; BPRS, Brief Psychiatric Rating Scale; SD, standard deviation.

Table 2 Informed, Met and Unmet Needs (CAN User's Section I)

\begin{tabular}{|c|c|c|c|c|}
\hline $\begin{array}{l}\text { CAN Items } \\
(N=150)\end{array}$ & $\begin{array}{c}\text { Total Needs } \\
\text { N (\%) }\end{array}$ & $\begin{array}{c}\text { Met Needs } \\
\text { N (\%) }\end{array}$ & $\begin{array}{c}\text { Unmet Needs } \\
\text { N (\%) }\end{array}$ & $\begin{array}{c}\text { Unmet Needs/Total } \\
\text { Needs (\%) }\end{array}$ \\
\hline Psychological distress & $95(63.3)$ & $56(37.3)$ & $39(26)$ & 41 \\
\hline Daytime activities & $91(60.7)$ & $33(22)$ & $58(38.7)$ & 63 \\
\hline Information & $90(60)$ & $67(44.7)$ & $23(15.3)$ & 25 \\
\hline Psychotic symptoms & $83(59.9)$ & $64(47.2)$ & $19(12.7)$ & 22 \\
\hline Company & $80(53.4)$ & $19(12.7)$ & $61(40.7)$ & 76 \\
\hline Looking after home & $74(49.4)$ & $64(42.7)$ & $10(6.7)$ & 13 \\
\hline Food & $71(47.3)$ & $63(42)$ & $8(5.3)$ & II \\
\hline Intimate relationships & $70(46.7)$ & $4(2.7)$ & $66(44)$ & 94 \\
\hline Physical health & $60(40)$ & $49(32.7)$ & II (7.3) & 18 \\
\hline Accommodation & $57(38)$ & $42(28)$ & $15(10)$ & 26 \\
\hline Money & $53(35.4)$ & $46(30.7)$ & $7(4.7)$ & 13 \\
\hline Sexual expression & $49(32.6)$ & $2(1.3)$ & $47(31.3)$ & 95 \\
\hline Self-care & $47(31.3)$ & $4 \mathrm{I}(27.3)$ & $6(4)$ & 12 \\
\hline Safety to self & $42(28)$ & $25(16.7)$ & $17(11.3)$ & 40 \\
\hline Benefits & $4 \mid(27.4)$ & $22(14.7)$ & $19(12.7)$ & 46 \\
\hline Alcohol & $34(22.7)$ & $18(12)$ & $16(10.7)$ & 47 \\
\hline Drugs & $33(22)$ & $16(10.7)$ & $17(11.3)$ & 51 \\
\hline Safety to others & $31(20.7)$ & $16(10.7)$ & $15(10)$ & 48 \\
\hline Dependents & $26(17.3)$ & $12(8)$ & $14(9.3)$ & 53 \\
\hline Transport & $22(14.7)$ & $12(8)$ & $10(6.7)$ & 45 \\
\hline Education & $22(14.6)$ & $11(7.3)$ & $11(7.3)$ & 50 \\
\hline Digital communication & $18(12)$ & $9(6)$ & $9(6)$ & 50 \\
\hline
\end{tabular}

Abbreviation: CAN, Camberwell Assessment of Needs.

A relationship was also found between the presence of three or more unmet needs and the following variables: marital status $(\mathrm{p}=0.018$; Married/partnered vs single;
$\mathrm{OR}=0.405 ; \mathrm{p}=0.028)$, employment status $(\mathrm{p}=0.009$; unemployed vs pensioner; $\mathrm{OR}=3.667 ; \mathrm{p}=0.002$ ), voluntary admission $\quad(\mathrm{OR}=2.480 ; \quad \mathrm{p}=0.032), \quad$ BPRS score 
Table 3 Perceived Needs, Help Received from Services and Family Members (CAN Sections 2 and 3), Adequacy of Help and Satisfaction with It (CAN Section 4)

\begin{tabular}{|c|c|c|c|c|c|}
\hline $\begin{array}{l}\text { CAN Items } \\
(N=\mid 50)\end{array}$ & $\begin{array}{l}\text { Moderate or High } \\
\text { Help Needed from } \\
\text { Services } \\
\text { N (\%) }\end{array}$ & $\begin{array}{c}\text { Satisfied } \\
\text { with Perceived } \\
\text { Help } \\
\text { N (\%) }\end{array}$ & $\begin{array}{l}\text { Receive the } \\
\text { Right Type of } \\
\text { Help } \\
\text { N (\%) }\end{array}$ & $\begin{array}{l}\text { Moderate or High } \\
\text { Help Received from } \\
\text { Relatives } \\
\text { N (\%) }\end{array}$ & $\begin{array}{l}\text { Moderate or High } \\
\text { Help Received from } \\
\text { Services } \\
\text { N (\%) }\end{array}$ \\
\hline Psychological distress & $55(36.7)$ & II (76.7) & IIS (76.7) & $34(22.7)$ & $17(11.3)$ \\
\hline Intimate relationships & $51(34)$ & $95(63.3)$ & $96(64)$ & $15(10)$ & $10(6.7)$ \\
\hline Information & $45(30)$ & $|2|(80.7)$ & $|2|(80.7)$ & $10(6.7)$ & $11(14)$ \\
\hline Psychotic symptoms & $44(29.4)$ & $126(84)$ & $126(84)$ & $35(23.3)$ & $22(14.7)$ \\
\hline Daytime activities & $43(28.6)$ & $95(63.3)$ & $95(63.3)$ & $21(14)$ & $20(13.3)$ \\
\hline Company & $42(28)$ & $95(63.3)$ & $97(64.7)$ & $68(45.3)$ & $20(13.4)$ \\
\hline Physical health & $4 \mid(27.3)$ & $140(93.3)$ & $140(93.3)$ & $24(16)$ & $38(25.3)$ \\
\hline Accommodation & $31(20.6)$ & 132 (88) & $133(88.7)$ & $46(30.7)$ & $19(12.7)$ \\
\hline Sexual expression & $30(20)$ & $109(72.7)$ & $109(72.7)$ & $6(4)$ & $6(4.1)$ \\
\hline Benefits & $30(20)$ & $132(88)$ & $132(88)$ & $15(10)$ & $20(13.3)$ \\
\hline Food & $26(17.3)$ & $139(92.7)$ & $139(92.7)$ & $66(44)$ & $22(14.7)$ \\
\hline Looking after home & $26(17.3)$ & $140(93.3)$ & $140(93.3)$ & $59(39.4)$ & $19(12.7)$ \\
\hline Safety to others & $19(12.6)$ & $138(92)$ & $138(92)$ & $8(5.4)$ & II (7.3) \\
\hline Drugs & $18(12)$ & 137 (9I.3) & 137 (9I.3) & $6(4)$ & $8(5.3)$ \\
\hline Money & $15(10)$ & $|4|(94)$ & $142(94.7)$ & $42(28)$ & $14(9.3)$ \\
\hline Alcohol & $14(9.4)$ & $135(90)$ & $135(90)$ & $5(3.4)$ & $5(3.3)$ \\
\hline Safety to self & $13(8.7)$ & $135(90)$ & $135(90)$ & $16(10.6)$ & $3(2)$ \\
\hline Dependents & $13(8.7)$ & | 37 (9|.3) & $136(90.7)$ & $18(12)$ & $3(2)$ \\
\hline Self-care & $10(6.7)$ & $144(96)$ & $144(96)$ & $19(12.7)$ & $6(4)$ \\
\hline Education & $8(5.3)$ & $138(92)$ & $138(92)$ & $\mathrm{I}(0.7)$ & $0(0)$ \\
\hline Digital communication & $5(3.3)$ & $143(95.3)$ & $144(96)$ & $7(4.7)$ & I $(0.7)$ \\
\hline Transport & $5(3.3)$ & $140(93.3)$ & $140(93.3)$ & $17(11.3)$ & I (0.7) \\
\hline
\end{tabular}

Note: Bold values indicate significant results.

Abbreviation: CAN, Camberwell Assessment of Needs.

$(\mathrm{OR}=1.044 ; \mathrm{p}=0.004)$ and PSP score $(\mathrm{OR}=0.979 ; p=$ 0.013). Within the BPRS factors, depressive symptoms $(\mathrm{OR}=1.155 ; p=0.001)$ and positive symptoms $(\mathrm{OR}=1.048$; $p=0.044)$ were associated to having more unmet needs. Results of the bivariate analysis is displayed in Table 1. The multivariate logistic regression model, which included marital status, employment status, legal status of admission and BPRS scores, explained $29.5 \%$ of the variance (Nagelkerke's $R^{2}$ : 0.295). Multivariate model is shown in Table 4.

\section{Discussion}

Inpatients with severe mental disorders reported a mean of eight perceived needs and three unmet needs during hospitalization. Intimate relationships, company, and daytime activities were the most prevalent unmet needs not adequately provided. A higher number of unmet needs were associated with more psychopathological symptoms, poorer functioning, worse socio-occupational integration and voluntary admission. These results highlight social and relationship areas as potential targets for interventions to improve functionality and reduce symptoms after discharge and could be useful to prevent hospitalization and readmissions. In fact, a previous study of our group associated the lack of psychological support from informal carers with the risk of readmission. ${ }^{5}$

Inpatients showed a higher number of perceived needs and unmet needs than community-based studies conducted in Spain and other countries. A study conducted by Zuñiga et al in Spanish outpatient population found a mean of one unmet need per patient. ${ }^{8}$ A study conducted in Australia also found a higher number of unmet needs in hospitalised patients and the same three categories of unmet needs as the most frequent. ${ }^{15}$ In the study of Zuniga et al with outpatients, company and daytime activities were the most frequently unmet needs along with psychological distress, but information, sexual expression and intimate relationships needs were less prevalent in their sample. ${ }^{8}$ Another Spanish study found company and intimate relationships as the most prevalent unmet 
Table 4 Variables Associated with 3 or More Unmet Needs Included in the Multiple Logistic Regression

\begin{tabular}{|c|c|c|c|c|}
\hline Dependent Variable $\geq 3$ Unmet Needs & Coefficient & Adjusted OR & $\mathbf{C l}$ & $p$ \\
\hline $\begin{array}{l}\text { Marital status } \\
\text { Married/partnered } \\
\text { Separated/divorced } \\
\text { Single (reference) }\end{array}$ & $\begin{array}{c}-1.104 \\
0.540\end{array}$ & $\begin{array}{l}0.331 \\
1.716\end{array}$ & $\begin{array}{l}(0.125-0.882) \\
(0.655-4.490)\end{array}$ & $\begin{array}{l}0.014 \\
0.027 \\
0.272\end{array}$ \\
\hline $\begin{array}{l}\text { Employment status } \\
\text { Working } \\
\text { Unemployed } \\
\text { Retired } \\
\text { Pensioner (reference) }\end{array}$ & $\begin{array}{c}0.417 \\
1.612 \\
-0.626\end{array}$ & $\begin{array}{l}1.517 \\
5.059 \\
0.535\end{array}$ & $\begin{array}{c}(0.502-4.586) \\
(1.968-13.008) \\
(0.104-2.744)\end{array}$ & $\begin{array}{l}\mathbf{0 . 0 0 4} \\
0.460 \\
\mathbf{0 . 0 0 1} \\
0.535\end{array}$ \\
\hline $\begin{array}{l}\text { Legal status of admission } \\
\text { Voluntary } \\
\text { Involuntary (reference) }\end{array}$ & 1.114 & 3.047 & $(1.148-8.093)$ & 0.025 \\
\hline BPRS & 0.048 & 1.049 & $(1.014-1.085)$ & 0.006 \\
\hline
\end{tabular}

Notes: Nagelkerke's R squared: 0.295 . Hosmer-Lemeshow test $=0.810$. Bold values indicate significant results.

Abbreviations: OR, odds ratio; $\mathrm{Cl}$, confidence interval.

needs in outpatients with schizophrenia. ${ }^{10}$ Nevertheless, inpatients in our study show a higher percentage of unmet needs in these areas. A study with recently discharged patients in the United Kingdom also reported similar findings with regard to needs, with psychological distress, daytime activities, company and information being the most frequent needs. ${ }^{40}$ Results regarding unmet needs were also similar to the present study, with company, psychological distress, and daytime activities being the most prevalent. Other studies also report that inpatients and outpatients predominantly have unmet psychosocial needs. ${ }^{41-43}$ In general, there are notable differences between European countries with regard to patient's unmet needs. ${ }^{23}$ A study comparing the needs of patients in Granada and Mannheim found that unmet needs were higher in the Spanish city especially with regard to occupation. ${ }^{25}$ Furthermore, some studies have reported that basic needs such as money or benefits are also frequently unmet. ${ }^{10,28,41,44}$ These results are probably related to a lower degree of development of community and patient care services in the countries of these studies. Overall, several studies show that social needs are less met than basic needs in patients with severe mental illness. $^{13,39}$

Regarding the factors associated with the presence of more unmet needs, other studies have found similar results. For example, a higher number of unmet needs have been associated with higher psychopathological symptomatology, ${ }^{45-47}$ unemployment, ${ }^{48,49}$ less intimate relationships, ${ }^{49}$ and lower functioning. ${ }^{46,50}$
Mental health diagnoses were not associated with the number of unmet needs in our analysis. As previous studies mainly focus on inpatients with psychosis, needs in other disorders have been less addressed. However, a study in recently discharged Scottish population found that non-psychotic group reported a higher number of needs and a higher level of unmet needs. ${ }^{40}$ Recently, other study in psychiatric inpatients in New York did not find an association between needs classes and mental health diagnoses. ${ }^{51}$ Our results support that mental health care planning should be based in evaluation of perceived needs and not diagnosis alone. ${ }^{52}$

Our analysis shows that patients admitted voluntarily presented more unmet needs. Our unit serves a very large area so admissions are restricted to seriously ill patients who are often involuntarily admitted. Voluntary admissions are scarce because volitional capacity is usually not preserved. Only patients with a higher level of social issues (no accommodation, money problem) or less support in the community but moderate symptoms may be voluntarily admitted. This result could be due to the design of our healthcare system and should be interpreted with caution.

Although the study has a large sample of inpatients assessed, some limitations should be considered when interpreting the results. This is a exploratory study; thus, causal relationships cannot be stablished between unmet needs and factors associated to them. Besides, the patient's perception has been the only one taken. As previous studies show, patients' perception does not always coincide with needs observed by professionals. ${ }^{53,54}$ Another 
concern is that the sample come from a single centre, so the results should be generalised with caution to other settings. These findings should be interpreted with additional caution, as the sample is heterogeneous in terms of diagnoses, patients' characteristics and time from admission to assessment. The model explains less than one-third of the variance of the total number of unmet needs. Although several variables were included in our analysis, some factors are still missing. Socioeconomic status, number of children, ethnicity and substance use disorders have been shown in other studies to have an impact in the number of needs. ${ }^{22,51}$ Adherence with treatment may also play a role in symptoms needs. Future research should address these variables in hospitalized patients.

\section{Conclusion}

This is the first study that estimates the perceived needs of a representative sample of psychiatry inpatients in a city of Spain and factors that influence the presence of unmet needs. These results show how certain areas, especially those related to social relationships and daily activities are not covered by services and are potential areas for improvement. Patients with resistant symptomatology, low functioning, single or separated, unemployed and demanding treatment from mental health services are the profile with the most unmet needs. Evaluating needs is essential for planning effective health and social systems that improve functionality of patients and reduces hospitalization. Understanding perceived needs by users may lead to design better discharge planning and communitybased care.

\section{Ethics Approval}

The study was conducted according to the guidelines of the Declaration of Helsinki, and approved by the Ethics Committee of Malaga Northeast.

\section{Informed Consent Statement}

Informed consent was obtained from all subjects prior to study commencement.

\section{Funding}

The research was supported by the Andalusian Government co-founded by Fondo Europeo de Desarrollo Regional - FEDER (Grant reference: PI-0338/08).

\section{Disclosure}

The authors report no conflicts of interest in this work.

\section{References}

1. Inspectorate GBD of HSS, Group GBSOSWS. Care Management and Assessment: Summary of Practice Guidance; 1991.

2. Brewin CR, Wing JK, Mangen SP, Brugha TS, MacCarthy B. Principles and practice of measuring needs in the long-term mentally ill: the MRC needs for care assessment. Psychol Med. 1987;17 (4):971-981. doi:10.1017/s0033291700000787

3. Priebe S, McCabe R, Bullenkamp J, et al. Structured patient-clinician communication and 1-year outcome in community mental healthcare: cluster randomised controlled trial. Br J Psychiatry. 2007;191 (NOV):420-426. doi:10.1192/bjp.bp.107.036939

4. Drukker M, van Os J, Bak M, À Campo J, Delespaul P. Systematic monitoring of needs for care and global outcomes in patients with severe mental illness. BMC Psychiatry. 2010;10(1):36. doi:10.1186/ 1471-244X-10-36

5. Guzman-Parra J, Moreno-Küstner B, Rivas F, et al. Hospital readmissions in patients with severe mental illness. Community Ment Health J. 2018;54(2):189-196. doi:10.1007/s10597-017-0095-x

6. Slade M, Leese M, Taylor R, Thornicroft G. The association between needs and quality of life in an epidemiologically representative sample of people with psychosis. Acta Psychiatr Scand. 1999;100 (2):149-157. doi:10.1111/j.1600-0447.1999.tb10836.x

7. Sartorius N. Mental health needs, 2015: changes of concepts and consequences. Psychiatry Clin Neurosci. 2015;69(9):509-511. doi: $10.1111 /$ pcn. 12278

8. Zúñiga A, Navarro JB, Lago P, Olivas F, Muray E, Crespo M. Evaluation of needs among patients with severe mental illness. A community study. Actas Esp Psiquiatr. 2013;41(2):115-121.

9. Ochoa S, Haro JM, Autonell J, Pendas A, Teba F, Marquez M. Met and unmet needs of schizophrenia patients in a Spanish sample. Schizophr Bull. 2003;29(2):201-210.

10. Tomita A, Burns JK, King H, et al. Beyond symptom management: family relations, unmet needs of persons living with severe mental illnesses, and potential implications for social work in South Africa. Soc Work Health Care. 2016;1389:(January):1-16. doi:10.1080/ 00981389.2015.1088926

11. Brunt D, Hansson L. Comparison of user assessed needs for care between psychiatric inpatients and supported community residents. Scand J Caring Sci. 2002;16(4):406-413. doi:10.1046/j.14716712.2002.00085.x

12. Hayward M, Slade M, Moran PA. Personality disorders and unmet needs among psychiatric inpatients. Psychiatr Serv. 2006;57 (4):538-543. doi:10.1176/appi.ps.57.4.538

13. Grinshpoon A, Ponizovsky AM. The relationships between need profiles, clinical symptoms, functioning and the well-being of inpatients with severe mental disorders. J Eval Clin Pract. 2008;14 (2):218-225. doi:10.1111/j.1365-2753.2007.00836.x

14. Broadbent E, Kydd R, Sanders D, Vanderpyl J. Unmet needs and treatment seeking in high users of mental health services: role of illness perceptions. Aust N Z J Psychiatry. 2008;42(2):147-153. doi:10.1080/00048670701787503

15. Cleary M, Freeman A, Hunt GE, Walter G. Patient and carer perceptions of need and associations with care-giving burden in an integrated adult mental health service. Soc Psychiatry Psychiatr Epidemiol. 2006;41(3):208-214. doi:10.1007/s00127005-0017-z

16. Stobbe J, Wierdsma AI, Kok RM, Kroon H, Depla M, Mulder CL. Decrease in unmet needs contributes to improved motivation for treatment in elderly patients with severe mental illness. Soc Psychiatry Psychiatr Epidemiol. 2015;50(1):125-132. doi:10.1007/ s00127-014-0918-9

17. Werner S. Subjective well-being, hope, and needs of individuals with serious mental illness. Psychiatry Res. 2012;196(2-3):214-219. doi:10.1016/j.psychres.2011.10.012 
18. Lasalvia A, Bonetto C, Malchiodi F, et al. Listening to patients' needs to improve their subjective quality of life. Psychol Med. 2005;35 (11):1655-1665. doi:10.1017/S0033291705005611

19. Kortrijk HE, Kamperman AM, Mulder CL. Changes in individual needs for care and quality of life in Assertive Community Treatment patients: an observational study. BMC Psychiatry. 2014;14(1):306. doi:10.1186/s12888-014-0306-8

20. Slade M, Leese M, Ruggeri M, Kuipers E, Tansella M, Thornicroft G. Does meeting needs improve quality of life? Psychother Psychosom. 2004;73(3):183-189. doi:10.1159/00007 6456

21. Schmidt M, Ekstrand J, Tops AB. Self-reported needs for care, support and treatment of persons who frequently visit psychiatric emergency rooms in Sweden. Issues Ment Health Nurs. 2018;39 (9):738-745. doi:10.1080/01612840.2018.1481471

22. Sirotich F, Durbin A, Durbin J. Examining the need profiles of patients with multiple emergency department visits for mental health reasons: a cross-sectional study. Soc Psychiatry Psychiatr Epidemiol. 2016;51(5):777-786. doi:10.1007/s00127-016-1188-5

23. McCrone P, Leese M, Thornicroft G, et al. A comparison of needs of patients with schizophrenia in five European countries: the EPSILON study. Acta Psychiatr Scand. 2001;103(5):370-379. doi:10.1034/ j.1600-0447.2001.00207.x

24. Najim H, McCrone P. The Camberwell Assessment of Need: comparison of assessments by staff and patients in an inner-city and a semi-rural community area. Psychiatr Bull U K. 2005;29(1):13-17. doi:10.1192/pb.29.1.13

25. Salize HJ, Küstner BM, Torres-Gonzalez F, Reinhard I, Jfj E, Rössler W. Needs for care and effectiveness of mental health care provision for schizophrenic patients in two European regions: a comparison between Granada (Spain) and Mannheim (Germany). Acta Psychiatr Scand. 2007;100(5):328-334. doi:10.1111/j.16000447.1999.tb10875.x

26. Stefanatou P, Giannouli E, Konstantakopoulos G, Vitoratou S, Mavreas V. Measuring the needs of mental health patients in Greece: reliability and validity of the Greek version of the Camberwell Assessment of Need. Int J Soc Psychiatry. 2014;60 (7):662-671. doi:10.1177/0020764013511793

27. Saldivia S, Torres-González F, Runte-Geidel A, et al. Standardization of the MARISTÁN scale to measure needs in people with schizophrenia and related psychoses. Int J Soc Psychiatry. 2014;60 (3):219-226. doi:10.1177/0020764013481544

28. Kulhara P, Avasthi A, Grover S, et al. Needs of Indian schizophrenia patients: an exploratory study from India. Soc Psychiatry Psychiatr Epidemiol. 2010;45(8):809-818. doi:10.1007/s00127009-0126-1

29. Desviat M. La reforma psiquiátrica 25 años después de la Ley General de Sanidad. Rev Esp Salud Pública. 2011;85(5):427-436. doi:10.1590/S1135-57272011000500002

30. Aizpuru F, Latorre K, Beroiz B, Arriba J, Mosquera F, BernalDelgado E. Variabilidad en la tasa de hospitalizaciones por problemas de salud mental en centros hospitalarios de agudos. Gac Sanit. 2008;3:199-216.

31. Saz-Parkinson Z, Medel A, Cediel-García P, Castellote J, Bouza C, Amate JM. Trends on schizophrenia admissions during the deinstitutionalisation process in Spain (1980-2004). Soc Psychiatry Psychiatr Epidemiol. 2011;46(11):1095-1101. doi:10.1007/s00127010-0289-9

32. Salisbury TT, Killaspy H, King M. The relationship between deinstitutionalization and quality of care in longer-term psychiatric and social care facilities in Europe: a cross-sectional study. Eur Psychiatry. 2017;42:95-102. doi:10.1016/j.eurpsy.2016.11.011

33. Phelan M, Slade M, Thornicroft G, et al. The Camberwell Assessment of Need: the validity and reliability of an instrument to assess the needs of people with severe mental illness. Br J Psychiatry J Ment Sci. 1995;167(5):589-595. doi:10.1192/bjp.167.5.589
34. Rosales Varo C, Torres González F, Luna Del Castillo J, Jiménez Estévez J, Martínez Montes G. Fiabilidad del instrumento de Evaluación de Necesidades de Camberwell (CAN) (Versión española del CAN Estudio de fiabilidad). Actas Esp Psiquiatr. 2002;30(2):99-104.

35. Nasrallah H, Morosini P, Gagnon DD. Reliability, validity and ability to detect change of the Personal and Social Performance scale in patients with stable schizophrenia. Psychiatry Res. 2008;161 (2):213-224. doi:10.1016/j.psychres.2007.11.012

36. Apiquian R, Elena Ulloa R, Herrera-Estrella M, et al. Validity of the Spanish version of the personal and social performance scale in schizophrenia. Schizophr Res. 2009;112(1-3):181-186. doi:10.1016/ j.schres.2009.03.028

37. Overall JE, Gorham DR. The brief psychiatric rating scale. Psychol Rep. 1962;10:799-812. doi:10.2466/pr0.1962.10.3.799

38. Peralta V, Cuesta MJ. Psychometric properties of the Positive and Negative Syndrome Scale (PANSS) in schizophrenia. Psychiatry Res. 1994;53(1):31-40. doi:10.1016/0165-1781(94)90093-0

39. Hensel JM, Banayan DJ, Cheng C, Langley J, Dewa CS. Client and key worker ratings of need in first-episode psychosis early intervention programmes. Early Interv Psychiatry. 2016;10(3):246-251. doi:10.1111/eip.12171

40. Simons L, Petch A. Needs assessment and discharge: a Scottish perspective. J Psychiatr Ment Health Nurs. 2002;9(4):435-445. doi:10.1046/j.1365-2850.2002.00506.x

41. Joska JA, Flisher AJ. Needs and services at an in-patient psychotherapy unit. Afr J Psychiatry. 2007;10(3):149-2156. doi:10.4314/ajpsy. v10i3.30247

42. Macpherson R, Varah M, Summerfield L, Foy C, Slade M. Staff and patient assessments of need in an epidemiologically representative sample of patients with psychosis - Staff and patient assessment of need. Soc Psychiatry Psychiatr Epidemiol. 2003;38(11):662-667. doi:10.1007/s00127-003-0669-5

43. Wiersma D. Needs of people with severe mental illness. Acta Psychiatr Scand. 2006;113(SUPPL. 429):115-119. doi:10.1111/ j.1600-0447.2005.00728.x

44. Zahid MA, Ohaeri JU. Clinical and psychosocial factors associated with needs for care: an Arab experience with a sample of treated community-dwelling persons with schizophrenia. Soc Psychiatry Psychiatr Epidemiol. 2013;48(2):313-323. doi:10.1007/s00127-0120514-9

45. Ritsner MS, Lisker A, Arbitman M, Grinshpoon A. Factor structure in the Camberwell assessment of need-patient version: the correlations with dimensions of illness, personality and quality of life of schizophrenia patients. Psychiatry Clin Neurosci. 2012;66 (6):499-507. doi:10.1111/j.1440-1819.2012.02383.x

46. Ruggeri M, Leese M, Slade M, Bonizzato P, Fontecedro L, Tansella M. Demographic, clinical, social and service variables associated with higher needs for care in community psychiatric service patients - The south Verona outcome project 8. Soc Psychiatry Psychiatr Epidemiol. 2004;39(1):60-68. doi:10.1007/s00127-004-0705-0

47. Van Os J, Lataster T, Delespaul P, Wichers M, Myin-Germeys I. Evidence that a psychopathology interactome has diagnostic value, predicting clinical needs: an experience sampling study. PLoS One. 2014;9:1. doi:10.1371/journal.pone.0086652

48. Joska J, Flisher AJ. The assessment of need for mental health services. Soc Psychiatry Psychiatr Epidemiol. 2005;40:529-539. doi:10.1007/s00127-005-0920-3

49. Lefebvre J, Cyr M, Lesage A, Fournier L, Toupin J. Unmet needs in the community: can existing services meet them? Acta Psychiatr Scand. 2000;102(1):65-70. doi:10.1034/j.1600-0447.2000.1020010 65. $\mathrm{x}$

50. Slade M, Beck A, Bindman J, Thornicroft G, Wright S. Routine clinical outcome measures for patients with severe mental illness: CANSAS and HoNOS. Br $J$ Psychiatry. 1999;174:404-408. doi:10.1192/bjp.174.5.404 
51. McDonald KL, Hoenig JM, Norman CC. Identifying profiles of need among psychiatric inpatients approaching discharge in New York City: a latent class analysis. Soc Psychiatry Psychiatr Epidemiol. 2021;56(1):63-73. doi:10.1007/s00127019-01817-4

52. McCrone P, Strathdee G. Needs not diagnosis: towards a more rational approach to community mental health resourcing in Britain. Int J Soc Psychiatry. 1994;40(2):79-86. doi:10.1177/0020764094 04000201
53. Wiersma D, Nienhuis FJ, Giel R, Slooff CJ. Stability and change in needs of patients with schizophrenic disorders: a 15- and 17-year followup from first onset of psychosis, and a comparison between "objective" and "subjective" assessments of needs for care. Soc Psychiatry Psychiatr Epidemiol. 1998;33(2):49-56. doi:10.1007/s001270050022

54. Slade M, Phelan M, Thornicroft G. A comparison of needs assessed by staff and by an epidemiologically representative sample of patients with psychosis. Psychol Med. 1998;28(3):543-550. doi:10.1017/ s0033291798006564

\section{Publish your work in this journal}

Neuropsychiatric Disease and Treatment is an international, peerreviewed journal of clinical therapeutics and pharmacology focusing on concise rapid reporting of clinical or pre-clinical studies on a range of neuropsychiatric and neurological disorders. This journal is indexed on PubMed Central, the 'PsycINFO' database and CAS, and is the official journal of The International Neuropsychiatric Association (INA). The manuscript management system is completely online and includes a very quick and fair peer-review system, which is all easy to use. Visit http://www.dovepress.com/testimonials.php to read real quotes from published authors. 